\title{
DESARROLLO Y EVALUACIÓN DE LOS PROCESOS DE RAZONAMIENTO COMPLEJO EN CIENCIAS
}

\author{
Fidel Antonio Cárdenas Salgado Ph. D.
}

\begin{abstract}
Despite the enhanced importance given by science educators and researchers to evaluation in sciences in the past, the search for better and more reliable assessing processes is nowadays a research priority.

Although the areas of science to be assessed have been generally accepted as including knowledge of concepts and facts, process skills, science thinking, problemsolving skills, abilities reeded to manipulate laboratoty equipment and the disposition of students to apply scientific knowledge, the impact of assessment on students, teachers and parents has not been overlooked. However, advances in cognitive psychology, science education and research on assessment on science are calling for new dimensions, such as complex reasoning, to be researched in the field.

Complex reasoning is a competence included as a desirable outcome of Science education in many Science curricula and is characterized by the following attributes: problem-solving, decision-making and critical and creative thinking. This paper presents a theoretical framework fon assessment in science involving the development of competencies through science education and emphasizes complex reasoning. A working model to categorize assessment tasks in complex reasoning is described and some of the main questions to be researched in the une are stated. The need for interdisciplinary work as well as close interaction with other unes of investigation has also been put forward.

It is the main objective of this line of nesearch to explore and propose more systematic assessment processes in science education to trace the evolution of students understandings and achievements mainly in secondary schools.
\end{abstract}

\section{Introducción}

Antes de centrar directamente la atención en la evaluación de los procesos de razonamiento complejo o en especial en el tema de la evaluación en ciencias, es conveniente abrir un espacio, así sea muy corto, para reflexionar sobre aspectos muy generales, pero no por eso carentes de importancia en la educación en ciencias; tal es el caso, por ejemplo, de la concepción de ciencia y la educación en ciencias como fundamento del desarrollo humano y económico. Estas son dos dimensiones importantes que influyen directa e indirectamente en el aprendizaje y en los aspectos a evaluar a lo largo de la ejecución de un curso y en toda la vida escolar del hombre.

\section{Una concepción de ciencia}

Algunas ideas acerca de la naturaleza de ja ciencia se pueden tomar del pensamiento de Schlesinger (1). Según él, es posible que a jo largo de la evolución de la especie humana haya habido y quizá aún haya, culturas que han aceptado o que acepten el

\footnotetext{
Profesor Asociado Departamento de Química. Universidad Pedagógica Nacional Programa Interinstitucional de Doctorado en Educación Área Educación en Ciencias Naturales.
} 
mundo y su devenir sin mucho o ningún esfuerzo por explicarlo o por hacerlo comprensible; sin embargo, hoy se admite que la naturaleza humana es de por si inquisitiva y que quiere ir mas allá de la mera aceptación de los acontecimientos.

En la actualidad, hay cierto consenso en el sentido de que no existe una sola forma de ver y explicar el mundo. Para algunos es caótico impredecible y por tanto imposible de conocer; para otros es misterioso y mágico, por consiguiente solo conocible por quienes alcanzan el dominio de lo mágico y finalmente, hay también quienes aceptan una mezcla de estas creencias, es decir, que parte del mundo es susceptible de conocer y explicar y que parte de él y su funcionamiento es inexplicable. Es dentro de esta última forma de ver donde parece ubicarse mejor la ciencia.

Dentro de este contexto, la ciencia hace relación a la búsqueda de explicaciones naturales y racionales para los fenómenos que se observan en el universo. Es el producto del desarrollo intelectual de una cultura, quizá el máximo exponente del desarrollo del pensamiento humano, que incorpora una forma particular de pensar y un cuerpo coherente de conocimientos producto del esfuerzo de muchas generaciones de científicos.

Como forma de aproximación a la búsqueda de explicaciones, la ciencia es intencionada, quienes laboran en ella lo hacen con propósitos específicos; es parcial, ningún científico conoce absolutamente todo; es orientada por una construcción teórica, lo cual significa que ninguna investigación científica parte de cero; es empírica, ya que trata de confrontar las representaciones intelectuales del mundo con la realidad; es social, en a medida que es una producción colectiva; es dinámica, puesto que sus explicaciones son falibles y por tanto modificables con el tiempo.

Por otra parte, la ciencia tiene una profunda repercusión social a nivel mundial; tal repercusión se puede observar en distintos ámbitos de la sociedad, en lo económico, en lo político, en lo ambiental, en lo ético yen lo tecnológico. Así, la educación en ciencias, y a través de las ciencias, ha de representar para cada estudiante un espectro de oportunidades que le permitan participar activamente en las empresas propias de la ciencia, es decir en la búsqueda de explicaciones en sus procesos de producción, en sus aplicaciones y en sus usos.

Es preciso, sin embargo, dejar claro que la ciencia no es un tipo de conocimiento arbitrario. Al igual que en las demás formas de conocimiento existen criterios de juicio que le son aplicables para establecer su validez, si bien cada forma de conocer tiene sus propios criterios de juicio, que no necesariamente son transferibles de un campo a otro, existe uno que es aplicable al conocimiento en todas sus formas: es el criterio de satisfacción de la comunidad que produce y mantiene esa forma particular de ver el mundo (1), esta afirmación, lejos de ser una aceptación casual y superficial, estar satisfecho con una explicación, dentro de este contexto significa ubicar dentro de un constructo particular de explicaciones no solamente nuestra experiencia sino también nuestros valores y nuestras aspiraciones,

\section{La educación en ciencias base de un desarrollo humano y económico}

Considerando la educación en general como un proceso mediante el cual intencionadamente una sociedad, por una parte desarrolla pertenencia a su cultura en los nuevos ciudadanos y por otra contribuye a que cada uno de ellos mejore y desarrolle todas las potencialidades que sean susceptibles de hacerlo, la educación en ciencias 
puede considerarse como un proceso intencionado, mediante el cual desde las ciencias y a través de ellas, una sociedad contribuye a que cada ciudadano desarrolle y mejore todo aquello que en su naturaleza es susceptible de desarrollar y mejorar. Así, sí la educación es un medio de humanización, la educación en ciencias también lo es por extensión.

Si bien es cierto, que gran parte del acervo cultural de una sociedad se hereda de generación en generación por el simple hecho de nacer y crecer en ella, es el caso de muchas creencias y visiones religiosas del mundo o del conocimiento común, esta no es la situación de la herencia cultural científica de la humanidad. La educación en ciencias en una sociedad tiene sentido en la medida que se acepte y se juzgue importante e incluso necesario, que la juventud adquiera y practique ciertos conocimientos, habilidades, valores y perspectivas científicas las cuales no se adquieren solamente por el hecho de nacer y vivir dentro de esa sociedad. En otros términos, si se acepta, que para poder adquirir esas competencias se requiere de manera intencionada y sistemática exponer a los niños y a los jóvenes a una serie de actividades académicas a lo largo de su vida escolar, mediante las cuales muy probablemente se les facilita su adquisición (2).

Como base de un desarrollo humano que es, la educación en ciencias debe permitir a los ciudadanos el perfeccionamiento al máximo de todas sus capacidades y potencialidades humanas así como también una preparación adecuada para desempeñar un papel eficiente y digno dentro de ja sociedad, es decir que si algunos de ellos se dedican a la ciencia, a partir de ella puedan construir un proyecto de vida de calidad.

Por otra parte, en la mira de posibilitar el perfeccionamiento de los seres humanos desde la ciencia, es pertinente tener en cuenta que el conocimiento científico se produce y se desarrolla mediante el uso de habilidades características del hombre, dentro de las cuales se incluyen entre otras, habilidades practicas, de comunicación y de pensamiento; por lo tanto, es de esperarse que la exposición de los niños y de os jóvenes a diferente actividades científicas contribuyan a desarrollar dichas potencialidades

Como motor de desarrollo económico, la educación en ciencias ha de conducir a la formación de ciudadanos competitivos, que a su vez forjen sociedades competitivas. Las grandes organizaciones, las grandes compañías y en general las sociedades del mundo actual lachan y se preocupan por mantener una posición de liderazgo en un mundo cada vez mas basado en la capacidad de competir. A su vez, esta capacidad para competir está dependiendo en gran medida de los conocimientos científicos y tecnológicos que posean los individuos y las sociedades Es dentro de este contexto que el desarrollo de las potencialidades humanas debe estar orientado a preparar un ciudadano capaz de actuar con libertad, responsabilidad, pertenencia y reconocimiento

En una sociedad cimentada sobre los avances científicos y tecnológicos o que se mueva en esta dirección, el analfabetismo científico es excluyente; aquellas personas sin una visión científica del mundo y sin unos conocimientos científicos mínimos, no solamente tienen cada vez menos posibilidad de acceso al trabajo y por tanto a las fuentes económicas, sino también una poca participación activa en los diferentes ámbitos de la dinámica social. Desde este punto de vista un desarrollo económico que propenda por la igualdad social presupone una alta educación en ciencias con el fin de evitar o por lo menos mitigar el surgimiento de una nueva forma de discriminación social.

Una sólida educación en ciencias contribuye también a mantener la cohesión y la estructura social, así como también a mejorar la comunicación dentro de una cultura y entre las culturas. En la medida en que todos los miembros de una comunidad, profesen y 
practiquen formas comunes de entender el mundo, la vida, el entorno y por tanto tengan una idea clara de su existencia y de su razón de ser, se facilita la comunicación y la convivencia pacífica. Del mismo modo, la comunicación intercultural hoy por hoy se fundamenta en conceptos científicos o en sus aplicaciones tecnológicas.

\section{ALGUNAS CAPACIDADES HUMANAS SUSCEPTIBLES DE SER DESARROLLADAS DESDE LAS CIENCIAS NATURALES}

Seria muy difícil hacer una descripción detallada de todas y cada una de las capacidades ${ }^{1}$ humanas que se pueden desarrollar desde la educación en ciencias a lo largo de la vida escolar, sin embargo, a partir de la literatura acerca de los objetivos de la enseñanza de las ciencias y de los tópicos que se tienen en cuenta para evaluar, es posible encontrar que existen puntos característicos los cuales, bajo diferentes nombres se han buscado desarrollar en los estudiantes a través del tiempo. Además, a juicio de muchos profesores del área, estos aspectos no solamente aparecen como deseables sino también necesarios para la educación de jos seres humanos dentro de una sociedad.

De una manera muy esquemática, la educación en ciencias propende por el desarrollo de los siguientes grupos de capacidades a lo largo de la vida escolar de los alumnos (3)

\section{Habilidades Básicas - Habilidades de Procedimiento - Habilidades Investigativas}

Dentro de cada uno de estos grandes grupos de habilidades a su vez es posible ubicar subgrupos particulares. Así por ejemplo, entre las habilidades básicas se encuentran las de observar, recolectar datos, medir, manipular instrumentos, seleccionar apropiadamente metodologías particulares, interpretar adecuada y correctamente textos e instrucciones sencillas, así como también la habilidad requerida para seguir estas últimas en una forma eficiente.

Las habilidades procedimentales, que bien podrían llamarse también metodológicas comprenden las habilidades para hacer inferencias y las de seguir procedimientos propiamente dichas. Entre las habilidades de inferencia se pueden incluir: la distinción entre una observación y una inferencia, la elaboración de generalizaciones a partir de las observaciones y de otras inferencias, la realización de deducciones a partir de las hipótesis y la modificación de éstas para dar cabida a nuevas observaciones y a nuevos datos.

Entre las habilidades procedimentales se encuentran otras como: la adopción de métodos apropiados junto con los respectivos parámetros de seguridad para su aplicación en el desarrollo de un experimento determinado o de una investigación en proceso; la selección de procedimientos e instrumentos adecuados para la solución de un problema particular. En este grupo de habilidades también se incluyen aspectos como la capacidad para seleccionar apropiadamente los intervalos necesarios en una escala, durante la realización de mediciones particulares de una propiedad de la materia con instrumentos determinados, y el establecimiento de las variables propias acerca de las cuales se debe recoger información, una vez seleccionado un proceso especifico, así como también la recolección de los datos relacionados con estas variables y su presentación en forma adecuada.

\footnotetext{
${ }^{1}$ El término capacidad, dentro de este contexto, significa talento o disposición para la comprensión de los conceptos y procedimientos científicos y es, por lo tanto sinónimo de habilidad intelectual
} 
La habilidades investigativas por su parte, implican el domino de conocimientos en un área del saber, el domino de los procedimientos científicos, el pensamiento crítico y el razonamiento complejo entre otras $(4,5)$. El término razonamiento complejo incluye la resolución de problemas, la toma de decisiones y el pensamiento creativo (6).

Por otra parte, a través de la educación en ciencias es posible desarrollar algunas habilidades propias del dominio afectivo,, tales como la capacidad de emitir juicios de valor, el respeto por la forma de pensar de las demás personas, la capacidad de trabajo en grupo y por tanto la tolerancia y la convivencia social.

Sería presuntuoso pensar que en la descripción anterior están incluidas todas las potencialidades humanas que se pueden desarrollar desde la educación en ciencias, oque ésta es la mejor categorización de las mismas. A pesar de los esfuerzos investigativos que se adelantan en este campo, aún hay mucho por hacer. El desarrollo de otras potencialidades en el ser humano a partir de la educación en química, por ejemplo, todavía está por investigarse y es en este campo, como en el del establecimiento de otras categorías y formas de evaluarlas, donde se requieren los aportes de nuevas investigaciones.

\section{Metas de la Educación en Ciencias}

En última instancia la Educación en ciencias ha de ser un proceso a través del cual se forme un ciudadano en los aspectos profesionales y personales. Tanto la formación profesional como la personal deben orientarse hacia una utilidad individual y colectiva.

Así, la Educación en Ciencias debe apoyar a los estudiantes en el desarrollo de sus habilidades básicas, de procedimiento y de investigación a fin de formar hombres idóneos para vivir en sociedad.

A nivel un poco más particular, la educación en ciencias debe propender por la adquisición de la capacidad para recordar y aplicar conocimientos específicos, llevar a cabo procedimientos científicos y razonar en forma compleja, operar con eficiencia y seguridad materiales y equipos de laboratorio; desarrollar actitudes y valores de respeto y tolerancia para con sigo mismo, con los demás y con el medio ambiente. De la misma manera, la educación en ciencias debe formar para el dominio del lenguaje de la ciencia, la comunicación intercultural y por tanto para la adaptación a las situaciones cambiantes del mundo moderno.

\section{Educación en Ciencias y Adquisición de Competencias}

A lo largo del desarrollo de la educación en ciencias se han venido utilizando varios términos para denotar los resultados que se esperan obtener luego de un período escolar. Usualmente éstos términos están muy relacionados con las teorías del aprendizaje que fundamentan la acción del docente. De acuerdo con esas teorías, se han empleado vocablos como conductas, conocimientos, dominios, logros y habilidades, entre otros.

Desde hace ya algún tiempo se ha venido introduciendo con el mismo objetivo el término competencias, y hoy se habla del desarrollo de competencias, de la adquisición de competencias o de las competencias deseables que un estudiante debería tener al dejar la educación secundaria o la universidad. Así, durante la vida escolar la educación en ciencias propende por el mejoramiento de las habilidades básicas procedimentales e investigativas, cuyo fin último es hacerlo idóneo o apto para vivir en una sociedad. 
El término competencias dentro del contexto de resultados que se esperan de la acción educativa de las ciencias, describe la capacidad que posee un alumno para hacer uso de sus conocimientos y habilidades para lograr un propósito determinado (7).

Una concepción de competencias en el sentido de idoneidad o aptitud, para, de una parte, permite hacer énfasis en los resultados del aprendizaje, el cual debe tener un propósito y algún efecto que se pueda mostrar; y, de otra, estimula la evaluación de esos resultados deseables, ya que posibilita concentrar la atención en la evaluación, no sola mente de los propósitos que se quieren alcanzar, sino también de los conocimientos y de la pericia requerida para su empleo.

Si bien es cierto, que el concepto de competencias no puede verse aislado de los resultados que se esperan del sistema educativo en general y de la preparación requerida por un ciudadano para ejercer un rol determinado dentro de la sociedad, en el contexto de este documento se circunscribe su uso y significado al campo de la educación y en particular de la educación en ciencias. En estos términos es posible hablar de competencias esenciales o fundamentales, que pueden lograrse dentro de un área básica, de la educación, como son las ciencias naturales.

Una primera clasificación de estas competencias apareció por primera vez, en 1985, en la propuesta del comité de Calidad de a Educación en Australia en los siguientes términos Adquisición de información, comunicación de la misma, aplicación de procesos lógicos, desarrollo de tareas prácticas y de tareas en grupo. Algunos años más tarde, pudo llegarse a una caracterización un poco más sistemática de las competencias esenciales (8) que en la actualidad se buscan como resultados deseables de una creativa y eficiente educación en ciencias. Estas competencias se describen a comunicación:

Recolección análisis y organización de información. Se estima necesario que un ciudadano en la actualidad, desarrolle habilidades para ubicar, seleccionar y clasificar la información que se requiera en un momento determinado y presentarla de tal manera que sea útil para él mismo o para otras personas; de la misma manera, se requiere que sea competente para hacer una evaluación de la información en sí y de los métodos y fuentes empleadas para obtenerla, en otras palabras se requiere formar personas competentes para el manejo eficiente de información con alta calidad.

Comunicación de información e ideas. Una de las características del mundo moderno es la comunicación clara de la información y de las ideas y es preciso formar ciudadanos aptos para hacerlo, se trata del desarrollo de la capacidad de comunicarse con otras personas mediante todo un espectro de formas de comunicación que incluyen la expresión oral y escrita, el empleo de gráficas y otras formas no verbales de la misma.

Planeación y organización de actividades. No menos importante que las anteriores es el desarrollo individual de una gran capacidad de planeación y organización de sus propias actividades, lo cual incluye hacer un buen uso del tiempo y de los recursos, el establecimiento de prioridades y un continuo control de su propio desempeño.

Capacidad de trabajo en grupo. Se trata de ganar habilidad para interactuar con los demás en forma individual y grupal, cada vez más se requiere de personas con un alto sentido de pertenencia y una gran capacidad de trabajar en grupo con responsabilidad y eficiencia 
El empleo de ideas y técnicas matemáticas. En la sociedad moderna cada vez es más imperativa la necesidad de una gran idoneidad para manejar y aplicar ideas matemáticas para emplear el espacio, el tiempo y los números, así como también para la estimación de cantidades y para realizar aproximaciones dentro de contextos prácticos.

La resolución de problemas. Se propende también por el desarrollo de una gran competencia para aplicar estrategias de solución de problemas, no solamente en aquellos casos cuando el problema y la solución son evidentes, sino también en aquellas situaciones donde se requiere el pensamiento critico y una aproximación creativa a la situación para buscar un resultado.

El uso de la tecnología. El desempeño eficiente de un ciudadano en la sociedad actual requiere cada día más de una buena capacidad para hacer uso de la tecnología combinando sus habilidades físicas e intelectuales, para la operación de equipos con un claro entendimiento de los principios científicos y tecnológicos que se precisan para la exploración y la adaptación de nuevos sistemas

\section{Competencias y razonamiento complejo}

Del grupo de competencias descritas anteriormente, quizá, la que requiere un análisis más detallado acerca de sus relaciones con la educación en ciencias, es la de la resolución de problemas, por cuanto si bien es cierto que dentro de este campo se tratan problemas muy sencillos, lo es también, que la solución de problemas abarca el desarrollo de procesos de razonamiento muy complejos, como los que se requieren para tener éxito en la realización de una investigación; es quizá por esta razón, que en la actualidad se hace tanto énfasis en su desarrollo y en la investigación de formas adecuadas de establecer su progreso a través de los diferentes sistemas educativos.

Los antecedentes de la investigación en el campo del razonamiento complejo, al igual que los del establecimiento de competencias en los seres humanos, se ubican en los Estados Unidos en la década de los 80. Por aquella época, este movimiento, no solamente tuvo una gran influencia en la comunidad educativa, sino que desde allí se ha ido extendiendo a muchos otros países. En la década del 90 es una poderosa línea de investigación en el campo de la educación en ciencias en el mundo $(9,10)$

Implícitamente, la expresión razonamiento complejo conlleva una serie de habilidades de pensamiento entre las que se han identificado la resolución de problemas, la toma de decisiones, el pensamiento creativo y el pensamiento critico, que a manera de objetivos, propósito o resultados esperados, en la actualidad se expresan en los diferentes programas de las disciplinas científicas, para las distintas instituciones educativas del nivel secundario e incluso de la universidad $(3.11,12)$.

Dentro del contexto anterior, es posible decir que el domino de los procesos de razonamiento complejo implica el desarrollo de competencias para resolver problemas, tomar decisiones, hacer uso crítico de las teorías conceptos y principios científicos así como también, pensar en forma creativa acerca de ellos.

La habilidad para resolver problemas lleva, a su vez a ser capaz de: integrar los procedimientos o la información aprendida para dar cumplimiento a una tarea; combinar diferentes procesos científicos en una estrategia coherente para terminarla con éxito y responder con eficiencia a situaciones nuevas. 
En la toma de decisiones se incluye la capacidad para seleccionar apropiadamente los datos o conocimientos importantes junto con un procedimiento que permita alcanzar una conclusión

El pensamiento creativo, expresado en la capacidad para usar críticamente las teorías y los conocimientos científicos, a su vez implica ser capaz de: identificar las suposiciones sobre las cuales se hacen algunas generalizaciones, ubicar falacias aparentemente lógicas dentro de un argumento, reconocer conclusiones no válidas, establecer el valor de las ideas y la autoridad sobre la cual se hacen ciertas generalizaciones, críticamente examinar lo apropiado o no de los datos y demostrar originalidad en los diseños y en la producción relacionada con el desempeño.

Algunos otros estudios sobre los proceso de pensamiento complejo $(13,14,15,16)$ han conducido al establecimiento de las siguientes características que representan el grupo de atributos más utilizado para describir la resolución de problemas, la toma de decisiones, el pensamiento critico y el pensamiento creativo; nótese como este grupo de atributos incluye los descritos en los párrafos anteriores,

La solución de problemas. Implica ser capaz de identificar, reconocer y representar el problema, integrar información o procedimientos para alcanzar una solución y contrastar y analizar las soluciones obtenidas.

Toma de decisiones. Para tomar una decisión se requiere establecer una meta, un problema o un resultado; seleccionar conocimientos, información datos o procedimientos importantes que sean adecuados para llegar a una conclusión, a describir una situación o para sustentar un argumento; analizar información alternativa, datos o argumentos para hacer comparaciones y contrastes; emplear la inducción para hacer inferencias o predicciones que sean consistentes con un grupo de datos o suposiciones; seleccionar entre varias la mejor alternativa, emitir un juicio de valor; justificar la toma de una decisión con base en la información producida por si mismo o suministrada por otros y evaluar resultados a partir de una información dada

Pensamiento crítico y creativo. Se manifiesta en la idoneidad para identificar suposiciones con respecto a una información dada; sintetizar información; juzgar la credibilidad de una información, de unos datos y de la autoridad sobre las cuales se fundamentan; justificar un punto de vista con base en la información producida; hacer deducciones, es decir, sacar conclusiones que son verdaderas necesariamente si un grupo de suposiciones es verdadero; ubicar falacias lógicas dentro de un argumento; reconocer conclusiones que son invalidas; evaluar el valor de ideas o la utilidad de una información o dato y demostrar originalidad y propiedad en sus diseños producciones o desempeños.

\section{La evaluación del razonamiento complejo}

Autores como Barón (17), han propuesto para la evaluación de los procesos de razonamiento complejo de los estudiantes, técnicas tales como la discusión interactiva, el análisis de los escritos de los alumnos, la realización de diferentes actividades para observar el desempeño y las tradicionales pruebas de lápiz y papel; sin embargo, se ha argumentado con bases investigativas, que un reducido número de actividades desarrolladas en el aula no es criterio suficiente para juzgar la riqueza conceptual de un alumno en un momento dado (18). Además se cree que un sistema de evaluación basado en instrumentos que buscan prioritariamente establecer conocimientos declarativos, es 
poco congruente con el razonamiento complejo que trata fundamentalmente con procesos de pensamiento divergente.

Así pues, parece haber suficientes razones para dejar aparte las tradicionales pruebas de lápiz y papel o, por lo menos, disminuir su importancia y experimentar formas más abiertas incluyendo un aborde de aproximación múltiple.

Es pertinente, además, tener en cuenta que el desarrollo de los procesos de razonamiento complejo en un alumno, están acompañados de ciertas características actitudinales, cuyo control puede arrojar información importante para la evaluación de tales procesos (14). Algunas de estas manifestaciones de actitud son: un carácter reflexivo, una mentalidad abierta, la curiosidad, la perseverancia, la precisión y una gran capacidad de aceptar los distintos puntos de vista presentados por otras personas, es por ésta razón que dentro de ésta línea de investigación, la evaluación y el control de tales características es una parte importante.

\section{Un modelo para la clasificación de las tareas de razonamiento complejo}

Una de las situaciones a resolver, dentro del contexto de esta línea de investigación, es la relacionada con la categorización de una tarea determinada como resolución de problemas, pensamiento critico, pensamiento creativo o toma de decisiones. En este sentido, se han seguido varios caminos, el primero de ellos es mirar detenidamente las palabras con las cuales se presenta la tarea; otro consiste en identificar las pa labras o frases que subyacen en los constructos de cada caso. Sin embargo, las dos formas anteriores carecen de uniformidad en el establecimiento de categorías, ya que las palabras tienen diferentes sentidos para distintas personas.

En realidad lo que se requiere es un modelo que permita relacionar las tareas de evaluación y los constructos subyacentes con las categorías de razonamiento complejo definidas en los términos ya expuestos.

Una aproximación en la dirección anterior ha sido propuesta por Wakefield (19), cuyo modelo, con algunas modificaciones (20), resulta muy útil en el establecimiento de categorías para la evaluación de los procesos de razonamiento complejo, ya que es posible clasificar las tareas como cerradas o abiertas, según la presentación de sus términos iniciales y finales.

Cuando se ordenan las características de estas tareas y sus respuestas en forma continua, en un extremo las cerradas" y en otro las abiertas', y estos continuos se ubican sobre las coordenadas cartesianas, entonces se encuentra un campo de definiciones teóricas que se divide en los cuatro cuadrantes. Estos cuatro cuadrantes son: tareas cerradas con respuestas cerradas, tareas cerradas con respuestas abiertas, tareas abiertas con respuestas cerradas y tareas abiertas con respuestas abiertas. Estos cuatro cuadrantes pueden hacerse corresponder bastante bien con los cuatro procesos del razonamiento complejo; resolución de problemas, toma de decisiones, pensamiento crítico y pensamiento creativo, como se representa en la figura 1. 


\section{Respuestas Abiertas}

\begin{tabular}{|c|c|}
\hline $\begin{array}{l}\text { Toma de decisiones } \\
\text { - Pensamiento divergente } \\
\text { - Dominio en la solución de problemas } \\
\text { - Hacer inferencias }\end{array}$ & $\begin{array}{l}\text { Pensamiento creativo } \\
\text { - Pensamiento divergente } \\
\text { - Síntesis de información } \\
\text { - Demostrar originalidad }\end{array}$ \\
\hline $\begin{array}{l}\text { Tareas } \\
\text { Cerradas }\end{array}$ & $\begin{array}{l}\text { Tareas } \\
\text { Abiertas }\end{array}$ \\
\hline $\begin{array}{l}\text { Solución de problemas } \\
\text { - Pensamiento convergente } \\
\text { - Dominio de procesos y algorítmicos } \\
\text { - Razonamiento lógico y evaluativo }\end{array}$ & $\begin{array}{l}\text { Pensamiento critico } \\
\text { - Pensamiento convergente } \\
\text { - Conclusiones intrínsecas o inherentes } \\
\text { - Razonamiento deductivo }\end{array}$ \\
\hline
\end{tabular}

\section{Respuestas cerradas}

Figura 1. Un modelo para la categorización de tareas en los procesos de razonamiento complejo (19).

Puesto que en el modelo se involucran variables continuas y no discretas, la discusión sobre la ubicación de situaciones abiertas y cerradas es de tipo racional y contextual, por tanto, no puede esperarse una ubicación estática y única para cada una de las situaciones y su clasificación de alguna manera está sujeta al juicio de quien las juzgue.

Dicho de otra manera, las situaciones que involucran solución de problemas, toma de decisiones, pensamiento creativo y pensamiento crítico, no necesariamente están confinadas a una sola categoría, puede haber aspectos de otras categorías que acompañan a una predominante; así por ejemplo, una situación de resolución de problemas puede incluir algún grado de toma de decisiones o una situación de toma de decisiones puede también incluir algo de pensamiento critico, por supuesto que una situación de pensamiento critico también puede requerir del pensamiento creativo.

A pesar de todo y dentro de un contexto particular, las categorías de tareas y sus respuestas se pueden identificar en primer lugar ubicándolas como relativamente abiertas o relativamente cerradas; y en segundo lugar por los constructos constituyentes de razonamiento complejo que estén presentes en cada caso, de esta manera a partir de dicha clasificación es posible proponer los respectivos esquemas de calificación. A continuación se presenta una descripción más detallada para la interpretación del modelo

\section{Resolución de problemas: tareas cerradas y respuestas cerradas}

La tarea es cerrada porque presenta en forma bien definida o bien estructurada un estado inicial en el cual el conocimiento la información o los parámetros requeridos para 
responderla se presentan todos o se asumen que son conocidos La respuesta es cerrada ya que la tarea tiene bien definida una meta final, en otras palabras, la respuesta se produce a partir de un procedimiento general conocido y la solución se evalúa contra un parámetro o estándar establecido por acuerdo Así, la situación requiere de un pensamiento convergente o de un pensamiento lógico para producir una respuesta que sea aceptable para una situación particular. La connotación de problema cerrado en este caso es la misma presentada por Hadden y Johstone para el caso de los miniproyectos (21).

El siguiente ejemplo ilustra esta situación desde el campo de la química. En cuatro tubos de ensayo se tienen soluciones de Cloruro de Bario, Sulfato de Cobre, Ácido Sulfúrico diluido y Cloruro de Sodio. Sin ayuda de ningún otro reactivo químico, establezca en cual tubo de ensayo se encuentra cada una de las soluciones.

\section{Toma de decisiones: tarea cerrada y respuesta abierta}

En esta categoría se ubican aquellas situaciones donde la tarea tiene un estado inicial bien definido pero una meta final poco establecida. De esta manera, el problema está bien establecido pero sus soluciones son abiertas hasta el punto de que pueden tener muchas variaciones y todas ellas son aceptables. Dentro de estas situaciones se encuentran aquellos casos de estrategias de resolución de problemas o de pensamiento divergente, en donde se apunta a la utilización de la toma de decisiones.

En el caso de las ciencias biológicas, en este tipo de tareas puede incluirse la descripción de restos fósiles que se hayan encontrado, donde se incluyan respuestas concernientes a su posible nicho, hábitat y clasificación.

Desde el punto de vista de la Química, la situación anterior se puede ilustrar así: A partir de los siguientes reactivos químicos Zinc, Ácido Clorhídrico diluido, cinta de Magnesio, Clorato de Potasio, Dióxido de Manganeso e Hidróxido de Sodio, produzca diferentes reacciones químicas y clasifíquelas.

\section{Pensamiento crítico: tareas abiertas y respuestas cerradas}

La tarea es abierta porque tiene un bajo nivel de definición en su estado inicial o éste es poco estructurado. Esto es, comparada con aquellas tareas bien definidas, las señaladas poseen pocas indicaciones de procedimiento y pocos parámetros para construir la respuesta o solución. En estos casos la respuesta es cerrada puesto que se requieren procedimientos bien establecidos en cuya aplicación se precisa emplear el pensamiento convergente, Además, se requiere el reconocimiento y una selección interna de alguna estrategia de procedimiento que no es muy obvia.

Así por ejemplo, en aquellos casos en los cuales a partir de una poca información sobre una enfermedad se requiere su diagnostico y sus causas para recomendar un adecuado tratamiento, quien lo hace necesita una gran habilidad en el manejo del pensamiento critico para responder en forma adecuada, no necesariamente de la misma manera que lo harían otras personas.

Una situación semejante a la anterior se presenta con la siguiente tarea: Calcular el volumen molar de un gas a 18 grados centígrados y $560 \mathrm{~mm}$ de mercurio 


\section{Pensamiento creativo: tareas abiertas y respuestas abiertas}

En algunos casos extremos la tarea no tiene ninguna definición para el alumno y él la tiene que definir. Lo mismo sucede con la producción de la respectiva respuesta. El grado de apertura de la tarea varía de acuerdo con el contexto, con la misma tarea y con el nivel de empleo de estrategias de pensamiento creativo. Así por ejemplo, la propuesta de un método para estudiar prácticas de conservación y describir estrategias de manejo para optimizar el uso de un área de interés grupal, requieren un grado razonable de pensamiento creativo. De igual modo, el diseño de una actividad práctica que permita establecer los diferentes tipos de soluciones o de mezclas.

\section{El carácter interdependiente de esta línea}

Puesto que el proceso de aplicación de instrumentos de recolección de datos en una investigación, así como también su diseño y construcción obedecen a un determinado paradigma, habrá tantos instrumentos y modificaciones de los mismos, cuantos principios teóricos desde los cuales se oriente la investigación.

De cualquier manera, independientemente de la teoría desde la cual se enfoque cada trabajo, habrá una forma de control propia de la misma investigación y unos logros preestablecidos a alcanzar, el carácter autocorrectivo de la investigación así lo exige (22).

En estas condiciones, la línea de investigación en evaluación estará íntimamente ligada al paradigma o a los paradigmas teóricos que soporten el concepto de aprendizaje o de desempeño académico. Así, en cada uno de los proyectos de tesis se han de integrar conocimientos de disciplinas como la Sicología Cognitiva, las Ciencias Naturales, y la Educación en Ciencias, en este sentido serán de gran utilidad los logros y progresos de las demás líneas del programa

\section{PERSPECTIVAS DE LA LÍNEA}

Con todo lo difícil que resulta hacer predicciones sobre los rumbos futuros de una tarea que apenas comienza, en principio, los trabajos dentro de esta línea se orientan a determinar las concepciones de ciencia, evaluación, importancia de la ciencia y la evaluación, existentes actualmente en profesores y alumnos y a establecer el progreso de estos últimos en el logro de competencias dentro del contexto descrito en este documento. Naturalmente, la evaluación del progreso de los alumnos hacia el logro de una o varias competencias implica por parte de los investigadores el diseño y la experimentación de los instrumentos respectivos.

Por otra parte, los aspectos desarrollados en las páginas anteriores, permiten formular algunas preguntas, cuyas respuestas, a través de una o varias tesis, han de conducir a la consolidación de esta línea como un campo de producción de conocimiento que oriente y dirija la evaluación en ciencias en el país.

Una de estas preguntas hace relación con el pasado y la evolución de la evaluación en ciencias en Colombia, ¿cuál ha sido su pasado y su evolución?, la búsqueda de respuestas a esta pregunta permite tener claridad sobre los métodos y las competencias que se han evaluado y en la actualidad se están evaluando en ciencias, este conocimiento es fundamental para proyectar sobre bases sólidas las nuevas orientaciones de la evaluación en Ciencias. 
Otros de estos interrogantes pueden formularse de la siguiente manera: ¿cuáles son, si existen, los mejores instrumentos para evaluar las habilidades básicas procedimentales e investigativas en un grupo de estudiantes?, cuáles son las mejores formas de evaluar los procesos de razonamiento complejo en las ciencias naturales? Las respuestas a estas preguntas podrían conducir a la producción de materiales de evaluación de nivel general en el país así como también, al ofrecimiento de cursos de formación permanente de profesores que se interesen por el mejoramiento de los procesos de evaluación.

También es de gran importancia dentro de esta línea, la búsqueda de respuestas a preguntas como ¿cuáles son las concepciones actuales que los docentes tienen sobre habilidades básicas, procedimentales e investigativas? ¿Es posible o no, elaborar una matriz de relaciones entre las capacidades humanas a desarrollar y el papel educativo de las ciencias naturales? Y, quizá más importante aún tratar de responder a la pregunta ¿cuáles son las habilidades básicas, procedimentales e investigativas que poseen los docentes actuales de ciencias?

Dado que la educación colombiana actualmente transita de un sistema educativo a otro, y por tanto de un sistema evaluativo basado en las normas y la autoridad del Ministerio de Educación, hacia un sistema basado en la institución educativa y su medio, tiene mucho sentido la realización de una o varias investigaciones orientadas a diseñar y experimentar modelos de evaluación centrados en criterios propios de cada institución con base en su misión particular.

Toda la búsqueda de respuestas a estas preguntas y a muchas otras que se formulen los doctorandos alrededor de estos temas conducirán a establecer algunos de los lineamientos generales sobre la evaluación por logros que hoy tanto necesitan los docentes no sólo de ciencias sino de todas las áreas en el país.

\section{Referencias}

1. Schlesinger, Allen B. Explaining Life. McGraw-Hill, Inc. 1994.

2. The A.S.E. Scienceleachers Handbook. Edited by Jhon Nellist and Brian Nicholl. Hutchinson and Co.Ltd. 1986.

3. Board of Senior Secondary School Studies. Queensland. Chemistry Senior Syllabus. 1995.

4. Butler J. Teachers Judging Standards in Senior Science Subjects. Fifteen Years of the Queens Land Experiment. Studies in Science Education. 26. p 135-157. 1995.

5. Bicel K.G. , McGregor J., Robertson I. J. y Weston R. A. J. Techniques for Assessing Process Skills in Practical Science. TAPS II. Heineman Education Book. 1989.

6. Benderson A. Crítical Thinking: Critical Issues. Princenton N.J. Educational Testing Service. 1990.

7. karmel, P. Quality of Education in Australia. Report of The Quality of Educatién Review Committee. AGPS, Camberra. 1985. 
8. Mayer Committee. Employment Related key Competencies: A Proposal for Consultation. Mayer Committee Melburne. 1992.

9. Butler J. Classroom Strategies to Develop Complex Reas6ning Skills: Problem Solving, Decisiomn Making and Critical Thinking. The Queensland Science Teacher, 1993. vol 19 $\mathrm{N}^{\mathrm{a}}$ 4. P 12-33.

10. Lordan C.P. Assessing Complex Reasoning Processes in Biological Sciences in Queensland. Master of Sciences Thesis. Graduate School of Education. Queensland University. 1996.

11. Board of Senior Secondary School Studies. Queensland. Physics Senior Syllabus. 1995.

12. University of Queensland. Graduate School of Education. Science Area Curriculum Study .1996.

13. Edwards J. y DallAlba G. Development of a Scale of Cognitíve Demand for Analysis of Printed Secondary Science Materials. Research in Science Education. 11. 1981.

14. Ennis R.H. A Taxonomy of Critical Thinking. In J.Baron and R. Sternberg (Eds). Teacing Thinking Skills: Theory and Practice. New York. W.H. Freeman and Company.1987.

15. kean M. Assessing Higher Order Thinking Skills: An Overview of The Issues, Princeton, NJ: ERIC Clearinghouse on Tests, Measurement and Evaluatíon. 1986.

16. Quellmalz E.S. Developing Reasoning Skills. In J. Baron and R. Sternberg (Eds). Teacing Thinking Skills: Theory and Practice. New York W $\mathrm{H}$. Freeman and Company.1987

17. Baron J.B. Evaluating Thinking Skills in the Classrrom. In J.Baron and R. Sternberg (Eds).Teacing Thinking Skills: Theory and Practice. New York. W.H. Freeman and Company.1987.

18. White R. Implications of Recent Research on Learnig for Curriculumand Assessment Journal of Curriculum Studies. 24. 2. 1992.

19. Wakefield J. Creative Thinking: Problem Solving Skills and The Arts Orientation. Norwood, N. J: Ablesh Publishing Corporation. 1992.

20. Jordan P and Kerr C. The Getting of Wisdom: Assessing Critical Thinking In The Chemistry Classroom. Proceedings of The $14^{\text {th }}$ International Conference on Chemical Education. Workshop 2-12.The University of Qeensland. 14-19 July 1996. Brisbane Australia.

Editor:Warren F. Beasley.

21 Hadden B and Johnstone A. H. Miniprojects An Introducction to the "World of Scíence"? Chemeda. Australian Journal of Chemical Education. Vol. 27. Pp. 39-45. March 1990

22. Cohen L. and Manion L. Research Methods in Education.Third Ed. Routledge. London 1989. 